\title{
The Quest for Improved Reproducibility In MALDI Mass Spectrometry
}

\author{
Matthew B. O'Rourke ${ }^{1}$, Steven P. Djordjevic ${ }^{2}$ and Matthew P. Padula ${ }^{*}$ \\ 1: Proteomics Core Facility, University of Technology Sydney, Cnr Harris and Thomas St, Ultimo, \\ NSW, 2007 Australia \\ 2: The iThree Institute, University of Technology Sydney, Cnr Harris and Thomas St, Ultimo, NSW, \\ 2007 Australia
}

\section{Corresponding Author}

Matthew P Padula, Proteomics Core Facility, University of Technology Sydney, Cnr Harris and Thomas St, Ultimo, NSW, 2007 Australia. Phone: +61295148374, Email:

matthew.padula@uts.edu.au

Short Title: Improving Reproducibility in MALDI MS

Keywords: MALDI MS, Nitrocellulose, Matrix, Reproducibility.

Acknowledgements: The authors do not wish to acknowledge any additional contributors to this manuscript. 


\section{Table of Contents}

Abstract

I. Introduction

II. Standard MALDI sample Preparations
A. Seeding Method
B. Crystal doping method
C. Rapid crystallisation
D. Sandwich method
E. Co-Mixing method
F. Electrospray method

III. Properties of different matrices
A. Organic acid matrices
B. Ionic liquid matrices
C. Proton stripping matrices
D. Inorganic matrices

IV. Matrix Dopants and additives

V. Enhanced MALDI target plates

VI. Nitrocellulose

VII. Properties of Nitrocellulose

VIII. Current consensus of effectiveness of methodologies

IX. The application of low cost robotics and microfluidics to MALDI.

X. Post-acquisition processing of spectra to enhance signal.

XI. Conclusion 


\title{
The Quest for Improved Reproducibility In MALDI Mass Spectrometry
}

(1)

\author{
Matthew B. O'Rourke ${ }^{l}$, Steven P. Djordjevic ${ }^{2}$ and Matthew P. Padula ${ }^{1}$
}

\author{
1: Proteomics Core Facility, University of Technology Sydney, Cnr Harris and Thomas St, Ultimo, \\ NSW, 2007 Australia
}

2: The iThree Institute, University of Technology Sydney, Cnr Harris and Thomas St, Ultimo, NSW, 2007 Australia

Reproducibility has been one of the biggest hurdles faced when attempting to develop quantitative protocols for MALDI mass spectrometry. The heterogeneous nature of sample recrystallisation has made automated sample acquisition somewhat "hit and miss" with manual intervention needed to ensure that all sample spots have been analysed. In this review, we explore the last 30 years of literature and anecdotal evidence that has attempted to address and improve reproducibility in MALDI MS. Though many methods have been attempted, we have discovered a significant publication history surrounding the use of nitrocellulose as a substrate to improve homogeneity of crystal formation and therefore reproducibility. We therefore propose that this is the most promising avenue of research for developing a comprehensive and universal preparation protocol for quantitative MALDI MS analysis.

\section{Introduction}

Matrix Assisted Laser Desorption Ionisation Mass Spectrometry (MALDI MS) is a mass spectrometric technique first demonstrated by Karas and Hillencamp in the 1980's (Karas and Hillenkamp 1988). This technique is unique in that it allows for rapid analysis and greater throughput of a range of biomolecules compared to electrospray ionisation, with straightforward sample preparation. However, this technology also has some basic limitations in the fundamentals of its routine operation surrounding shot to shot reproducibility and the heterogeneous nature of spotted samples that have never been definitively resolved. These two parameters greatly affect quantitative reproducibility and the automation of the acquisition because of the presence of so called 'hot spots' or areas of high matrix:analyte concentration that have much greater ionisation efficiency than immediately adjacent points as close as $1 \mu \mathrm{m}$ away.

A great deal of effort has been dedicated over many years to improve the homogeneity of the matrix:analyte surface or spot and we present a review of a wide body of literature, methodological techniques and innovations, with a particular focus on the uses and applications of nitrocellulose, the only technique that has shown any level of reproducibility and surface consistency throughout a 30 year publication history. We also investigate proposed workflows for use with MALDI MS in an 
attempt to increase signal to noise $(\mathrm{S} / \mathrm{N})$, spot homogeneity and reproducibility during automated acquisition. We will also comment on the relative expense of these techniques with some innovations proving to be both low cost and easy to implement. While one could argue that the presently applied techniques are adequate for many applications, such as protein identification from in-gel enzymatic digests of electrophoretically separated proteins or nanoflow chromatography separated peptides, automated acquisition methods are somewhat 'hit and miss' affairs with manual re-acquisition often necessary. With the high sample throughput possible with MALDI, it only makes sense to improve the techniques robustness and throughput and remove the need for manual re-acquisition.

\section{Standard MALDI Sample Preparations}

Traditional MALDI sample preparation methods mostly use what is commonly known as the "dried droplet" (DD) method and it is a testament to the ease and robustness of this approach that it has been used almost unchanged since the mid-1990s. In the dried droplet method, a purified sample is spotted onto the surface of a metal or conductive target plate, allowed to dry before being overlayed with $1 \mu 1$ of an appropriate MALDI matrix(figure 1.)(Karas and Hillenkamp 1988). The DD method is by no means perfect, however it is the ubiquitous method for sample preparations involving whole cell lysates(Sedo, Sedlacek and Zdrahal 2011) and purified lipids(Bahr, Karas and Hillenkamp 1994), proteins(Sato et al. 2011), peptides(Zhao, Barber-Singh and Shippy 2004), metabolites(Weaver and Hummon 2013), drugs(Chughtai and Heeren 2010), DNA (Boom et al. 2004) and other organic molecules(Hillenkamp et al. 1991). The key problems with this method of sample preparation are the creation of regions of relative high sample intensity termed "Hot Spots" and the general poor spectral quality of analysing low concentration samples through the dilution effect of spreading the molecules of the same analyte across a relatively large area which is not completely sampled by the laser.

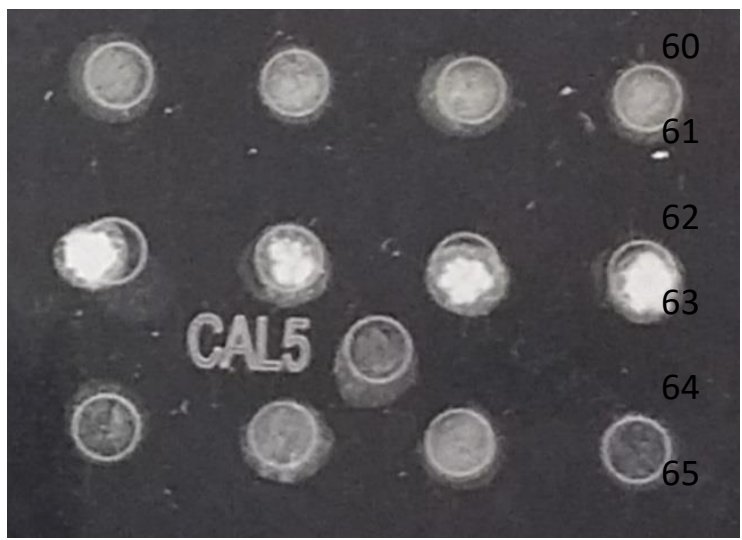

Figure 1. Dried droplet prepared samples. It can be clearly be seen that the middle row spots have crystallised unevenly resulting in a darker central "hotspot"

"Hot Spots" are caused by the uneven co-crystallisation of the matrix and analyte(T-W. Dominic Chan 1992). This lowers the protein concentration of the surrounding area making automated analysis "hit and miss" and forcing the user to manually search for these hot spots to acquire the highest intensity data(Nishikaze et al. 2012). This makes quantitation challenging as "hot spots" may contain levels of sample that cause ion suppression while other areas of the spot may not contain enough sample to generate sufficient signal to create a spectrum.

Low concentration samples also pose an issue in MALDI preparations as only $\sim 1 \mu$ of sample is used per spot which can cover a space $\sim 1 \mathrm{~mm}^{2}$, exponentially decreasing the concentration of the sample(Nordhoff, Lehrach and Gobom 2007). Samples can be concentrated by evaporation however for samples such as whole cell lysates, this has an added issue of increasing the concentrations of 
endogenous contaminants such as salt which will suppress ionisation in MALDI(Fountoulakis and Langen 1997).

There have been a number of variations of the DD method each aiming to improve either one or more of the following: reproducibility, signal to noise $(\mathrm{S} / \mathrm{N})$, spot homogeneity, the lower limit of detection and limit the uptake of contaminants. Each one is summarised briefly below:

A. Seeding method: A very thin layer of dilute matrix crystals is layered onto the surface of a target plate followed by overlaying with a mixture of the analyte and more matrix. This was reported to create homogenous spots(Onnerfjord et al. 1999) but adds an extra step requiring manual pipetting or robotics.

B. Crystal doping method: Also called the slow crystallisation method(Cohen and Chait 1996), it aims to reduce the uptake of contaminants during crystal formation and involves the dissolution of matrix crystals in solvent and water then slowly evaporating the surrounding liquid to allow long crystal shards (1-3 mm) to form, a process taking hours. The crystals are then individually selected, affixed to a MALDI target plate with liquid Styrofoam and analysed(Xiang and Beavis 1993).Numerous extra handling steps are therefore introduced as is the need for a skilled operator.

C. Rapid Crystallisation: This is reported to increase the ionisation efficiency of low mass peptides and involves spotting the sample followed by the matrix then placing the target plate into a vacuum chamber evacuated with a rotary pump. Crystallisation was observed to take $<20$ seconds(Cohen and Chait 1996).

D. Sandwich method: This method involves the layering of matrix then analyte then additional matrix allowing each stage to dry before adding the next. This is reported to increase minimum level of detection significantly with detection down to attomolar range being achievable with routine samples(Li, Golding and Whittal 1996) at the cost of extra steps of manual pipetting or the need for robotics.

E. Co-Mixing method: Sample solution and matrix solution are mixed in a 1:1 ratio then spotted directly onto a metal target plate. This is designed to increase the incorporation of the analyte molecules into the matrix crystals thereby increasing signal intensity(Cohen and Chait 1996), however the need to work with high concentrations of sample in volumes of $<1 \mu 1$ adds a layer of complexity.

F. Electrospray method: An electrospray setup, similar to that of LCMS, is used to create ion plumes that deposit both sample and matrix onto the surface of a MALDI target plate(Axelsson et al. 1997). The benefit of this setup is reported to be an improvement compared to other implementations of the DD method however it should be noted that the researchers in this paper used the electrospray setup to mimic the sandwich, co-mixing and seeding methods of sample application. It is therefore unclear as to the actual mechanisms behind the reported improvement in signal intensity although visual inspection of the spot indicates a more homogeneous surface. The adaption of this technique to automated high throughput sample spotting and analysis is challenging with some kind of autosampler and concentration technique necessary to ensure small volumes prior to electrospraying. Our personal experience with this technique was that reproducibility was challenging and the technique was not suited to routine analysis (unpublished data).

The above list of matrix application methods is not a complete description of the methods currently in use as there are other techniques that are specialised to sample types other than purified in-solution biomolecules. The methods of matrix application needed for technologies such as MALDI imaging 
(IMS) are vastly different; they do however rely on the same principles of crystal size and the effective co-crystallisation of the analyte and matrix(Kang et al. 2011). The most common method of matrix application in IMS is the use of automated spraying apparatuses that apply wet matrix in a fine mist that then crystallise with the analyte as it dries(Enthaler et al. 2013). The dry samples are then analysed. Applying matrix in this way is easily controllable however there is generally some level of variation from sample to sample that is introduced by micro-delocalisation caused by the droplets of sprayed matrix.

At the time of writing, the authors of this review are the only team who have published papers pertaining to improving and ensuring reproducibility in IMS sample preparation, and these methods relied on the application of dry matrix via sublimation (figure 2). Methods of accounting for sample preparation variation traditionally rely on post processing techniques that account for variation and normalise data accordingly to generate visually consistent images. This will be discussed further in section X.
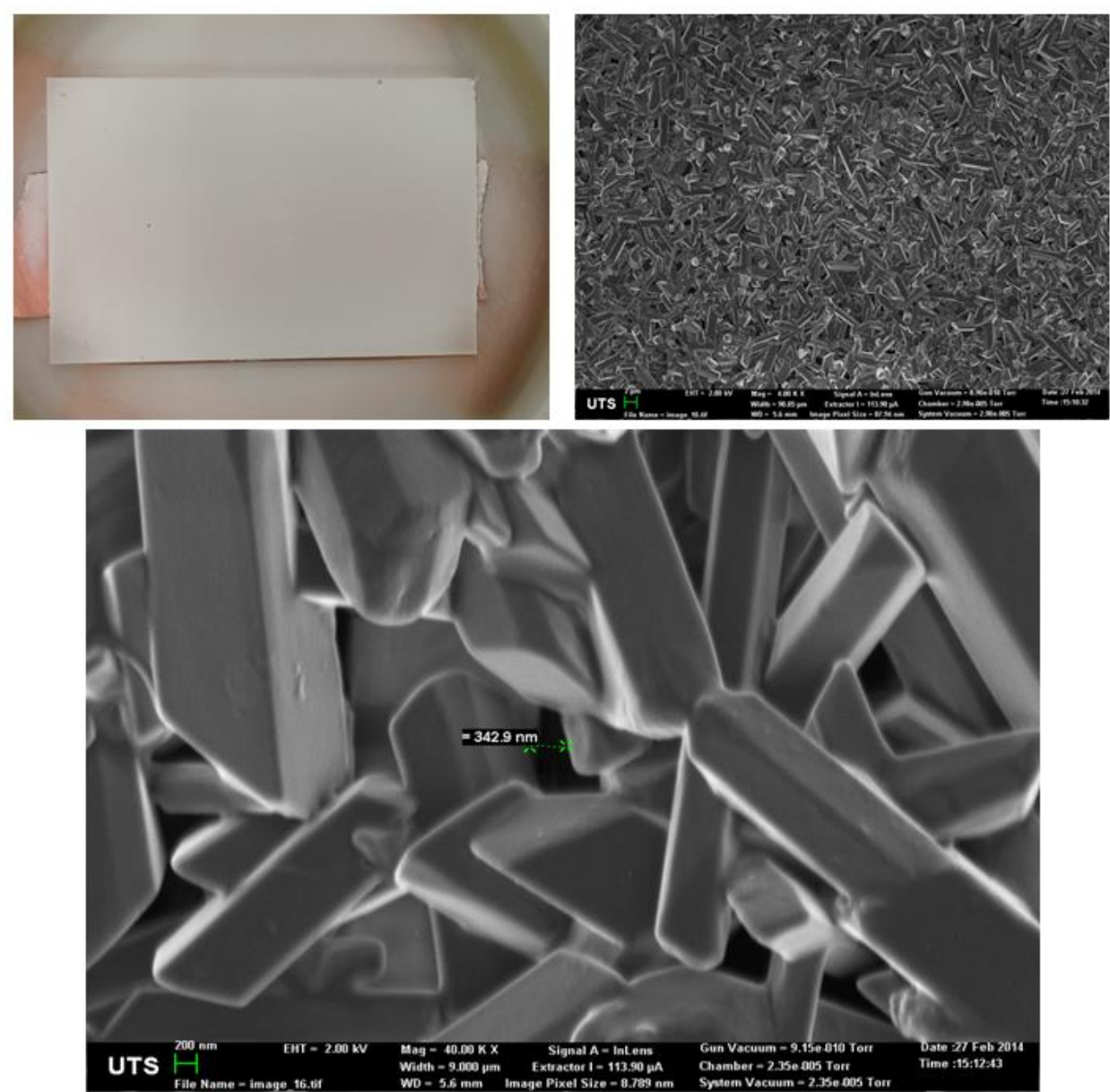

Figure 2. Sublimated matrix at increasing magnifications. Top left: sublimated matrix on an Indium tin oxide (ITO) coated glass slide showing a homogenous coating. Top Right: Scanning electron micrograph showing homogenous crystal formation at 4000x magnification. Bottom: 40000x magnification showing gaps between crystals of $350 \mathrm{~nm}$ 
The success of the above techniques is varied with no single technique proving to be more effective than any other in practice, evidenced by the still widespread use of the dried droplet method in spite of the existence of other techniques. There is still a great need to find a universal protocol to standardise MALDI sample application that improves reproducibility and is easy to implement. Variation in the method of sample and matrix application has not provided the answer, so attention is turned to the use of different and specific MALDI matrices for different sample types.

\section{Properties of different matrices}

MALDI, by definition, requires the use of a matrix that is able to absorb UV energy and then impart this energy to the analyte to excite the matrix and sample molecules and create an ion plume (Knochenmuss and Zenobi 2003). The choice of which matrix should be used is governed by a number of key factors, namely the sample type i.e. lipids, proteins, peptides etc. and the desire to run in either negative or positive mode. The next consideration is the secondary properties of each matrix including chemical modifications the matrix could make to the analyte and the nature and rate of cocrystallisation with the sample. It should be stated that the publications concerned with increasing signal intensity through the specialised selection of matrix were not overly concerned with whether the matrix was able to improve homogeneity during recrystallisation. It is our summation that the varied methods of matrix spotting mentioned above can be applied to any number of matrices and therefore specialised selection has been investigated for the specific purpose of increasing ion/signal intensity for specific samples.

MALDI matrices can be easily divided into four distinct classes: Organic acid, ionic liquid, proton stripping and inorganic, with each matrix type possessing distinct characteristics.

A. Organic acid matrices: these are the most commonly used matrices, defined as having an aromatic ring combined with an acidic side chain(Fukuyama 2015) which allows for the absorption of $\mathrm{UV}$ energy and donation of $\mathrm{H}^{+}$to the analyte although high concentrations of trifluoroacetic acid are also employed for this purpose. Organic acid matrices also have a wide range of uses and can induce chemical modifications in the sample(Krasny, Hynek and Kodicek 2011). They are also specific by nature with each matrix having an ideal sample type. For example, the matrix alphacyanohydroxycinnaminic acid (CHCA) is used preferentially for peptides(Bruker 2013) while sinapinic acid is used primarily for proteins (>3 kDa)(Bruker 2013) For a comprehensive review on the application on different types of matrices please see the excellent review by Fukuyama(Fukuyama 2015)

The biggest challenge faced when using solid organic acids, is the dissolution and recrystallisation of the matrix powder with the analyte. Recrystallisation generally produces large crystals with a high heterogeneity in size, shape and degree of coverage of the target plate surface. As the analyte is incorporated into the matrix crystals this directly contributes to the generation of hot spots. An example of this is the use of dihydroxybenzoic acid which forms long sharp crystals that border the pipetted spot(Fukuyama 2015). This leaves a void in the middle of the spot where sample ionisation is very poor, irrespective of the presence or absence of analyte molecules. Strategies that have been employed to try and solve this include those methods mentioned above in "Standard MALDI sample preparation" for dried droplet method of application. In addition to improving homogeneity, the ionisation efficiency of a specific analyte can be greatly improved through the specific choice of matrix. 
A study conducted by Frances et al in 2013(Francese et al. 2013) compared the use of a turmeric extract (curcumin) to the known matrix CHCA for use as new MALDI matrix in the analysis of lipids from latent fingerprints. The sample preparation for both of these matrices was standardised and they found that the success of each matrix was ion specific with CHCA generating high ion yields from diacylglycerol and curcumin being able to ionise glycerophosphocholine whereas CHCA did not. They also found that both matrices ionised oleic acid equally. Another approach that has been explored is the chemical modification of pre-existing matrices such as the chlorination of CHCA. Jaskolla et al explored the effects of this simple modification and concluded a preference for ionisation of peptides with a high $\mathrm{pH}(\mathrm{Jaskolla}$ et al. 2009).

B. Ionic liquid matrices: Ionic liquid matrices (ILC's) are a class of MALDI matrix that is stable in liquid form at room temperature and also while under vacuum. First described for use in MALDI by Armstrong et al in 2001(Armstrong et al. 2001), they are reported to significantly improve the homogeneity of analyte distribution as the matrix remains in a liquid state. The greatest benefit to this is the removal of the presence of "hot spots". However, the rate of ionisation is somewhat changed as the volatility of the liquid under high vacuum can be unpredictable( $\mathrm{Li}$ and Gross 2004). A high degree of specificity is also required when choosing the correct liquid matrix for a given sample type. This was also reflected in the work by $\mathrm{Li}$ and Gross (2004)(Li and Gross 2004) who commented that the use of ILC's for use in quantitation using MALDI is viable so long as the calibrant used matches chemical activity of the analyte within each specific matrix.

C. Proton stripping matrices: Proton stripping matrices have the ability to absorb protons from the analyte to facilitate negative mode ionisation of the sample. Compounds such as 9-aminoacridine can be used without introducing matrix ions in the low molecular weight range which makes them ideal for the analysis of metabolites, lipids and drugs(Sun et al. 2007). A paper by Vaidyanathan and Goodacre (2007)(Vaidyanathan and Goodacre 2007) attempted to use 9-aminoacridine to develop a quantitative workflow for the analysis of metabolites using MALDI. They found that they were able to analyse different concentrations of individual metabolites from a complex mixture without encountering analyte ion suppression from matrix ions in the low molecular weight range. They did however, comment on the large standard deviation of detected quantities as a result of the heterogeneity of the co-crystallisation of the sample and matrix.

D. Inorganic matrices: Inorganic matrices and the addition of inorganic components to various MALDI matrices have a long history of use that begins with the paper by Tanaka et al in 1988 for which he was awarded the Nobel prize (Tanaka et al. 1988). This work focused on incorporating inorganic ultrafine cobalt powder ( $300 \AA$ diameter) into the sample to be analysed. It was determined that the cobalt allowed for far greater ionisation of the sample when compared to just glycerol. This idea of using inorganic compounds for MALDI has been taken further by the work of Dong et al in 2010(Dong et al. 2010) who proposed the use of graphene as a novel MALDI matrix for small molecules. By using this form of carbon, the team were able to ionise nucleotides and drugs in a very low mass range ( $<300 \mathrm{Da}$ ) without the interference usually seen by matrix ions. There was also an increase in ionisation efficiency when compared to the more common CHCA matrix. Inorganic matrices are mostly chosen for their ability to assist in the ionisation of small molecules. Since the compounds chosen are usually very small $(<100 \mathrm{Da})$, they eliminate the interference that is usually seen in the low mass range when using traditional MALDI matrices such as CHCA. Currently a range of compounds have been used as inorganic matrices including silica/CHCA(Fleith et al. 2014), graphite(Sunner, Dratz and Chen 1995), -naphthylethylenediamine dihydrochlorid (NEDC)(Hou et al. 2014), sulfur(Kruegel, Pavlov and Attygalle 2013), titanium dioxide anatase(Castro et al. 2008), tungsten oxide(Bernier, Wysocki and Dagan 2015), mesoporous 
tungsten titanate(Shan et al. 2007), gold nano-particles(Marsico et al. 2015) and two dimensional graphene(Friesen et al. 2015)

\section{Matrix dopants and additives}

In addition to the careful selection, modification, and combining of different matrices, matrices can also be "doped" with additional compounds to increase ionisation efficiency or remove contaminants. The first record of doping matrices to increase ionisation was reported by Tanaka et al (1988)(Tanaka et al. 1988) who used cobalt powder to enhance the action of glycerine as a MALDI matrix.

Additional compounds have also been added to matrices such as the addition of ammonium phosphate which was reported by Zhu and Papayannapolous in 2003(Zhu and Papayannopoulos 2003). The addition of this compound is reported to prevent the addition of sodium and potassium adducts to peptides and proteins during ionisation. Jackson et al in 2014 also noted that ammonium phosphate had the same effect in negative ion mode (Jackson et al. 2014). Another dopant is the addition of phosphoric acid to DHB matrix to increase the ionisation efficiency of phosphopeptides in a crude peptide mixtures(Kjellstrom and Jensen 2004).

\section{Enhanced MALDI target plates}

One of the sources of reduced homogeneity is the spreading of samples beyond the area ablated during automated acquisition. MALDI target plates typically have target spots etched into the plate surface indicating the area that will be sampled. However, poor pipetting and the reduced liquid surface tension of solutions containing high percentages of organic solvent mean that samples often spread beyond this 'boundary'. This effect has been reduced by the introduction of target plates with modified surfaces to stop liquid spreading beyond the boundary, such as Bruker's AnchorChip and $\mu$ Focus plates by Hudson Surface Technologies(Technology 2010). These plates aim to reduce the size of the sample spot and thus increase the concentration of the analyte at the point being ablated thus increasing $\mathrm{S} / \mathrm{N}$. These plates are still subject to 'hot spots' because they do not change the crystallisation properties of the sample. In contrast to commercially manufactured MALDI target plates, a simple preparation can also be performed using commercially available Scotch Guard and a standard stainless steel MALDI target(Owen et al. 2003). This creates a uniform hydrophobic surface that allows samples of high aqueous composition to bead rather than disperse on the target surface, whereas the AnchorChip and $\mu$ Focus plates have a discreet hydrophilic region surrounded by a hydrophobic region.

\section{Nitrocellulose}

Nitrocellulose (NC) is a nitrated polymer that is reported to possess a number of unique properties that have a great potential application in biomedical research. The trends that are in the current body of literature demonstrate clearly that there are two categories of investigation when using nitrocellulose; most papers can be easily divided between: methodologies that aim to increase shot to shot reproducibility and the homogeneity of matrix and analyte co-crystallisation and papers that focus on the protein/peptide capture ability of NC that allows for concentration of dilute samples and subsequent washings that remove soluble contaminants such as salt. In addition to these two discrete streams of investigation, a significant portion of the literature also reports that $\mathrm{NC}$ can enhance signal to noise ratios(Mock, Sutton and Cottrell 1992), decrease contamination of samples from metal ion adducts(Liu et al. 1995), remove matrix ions from a spectra(Donegan et al. 2004), withstand multiple consecutive analyses(Kouvonen et al. 2009), provide effective crystal seed layers when combined 
with matrix in solution phase(Landry, Lombardo and Smith 2000) and, when used as a precoated layer, provide a hydrophobic surface that concentrates samples into a smaller space(Miliotis et al. 2002). If all of these claims are accurate, then there is a real need to incorporate NC into MALDI applications. The following is a review of all of these claims and a comprehensive look at the benefits of NC use in MALDI.

\section{Properties of Nitrocellulose}

Some of the earliest publications that described the applications of NC focused on its use as a semi permeable membrane for extracting toxins from bacterial culture(Brown 1915, Ruffer and Crendiropoulo 1901). It was not until 1986 when Jonsson et al(Jonsson et al. 1986) used NC membrane as a substrate to capture protein for use in Plasma Desorption Mass Spectrometry (PDMS). It was noted that the samples could be washed after spotting on NC which removed contaminants and significantly enhanced the signal intensity of the PDMS. Two years later Wilk et al(Wilk et al. 1988) continued this work and it was found that NC, when added directly to the sample increased spot to spot reproducibility and increased spot homogeneity.

In 1992, Mock et al(Mock, Sutton and Cottrell 1992) reported further use of NC as a substrate on a LaserMat sample target (Finnigan), allowing the sample to be washed prior to matrix application. It should be noted that a key step that was mentioned involved the addition of the matrix in liquid phase followed by the immediate covering of the target with a glass cover slip. It was reported that this was necessary to allow the ACN in the matrix to extract the protein from the surface of the NC thereby recovering what had become bound to the substrate layer and significantly enhancing the detected signal. The next year, Preston et al(Preston, Murray and Russell 1993) reported the first investigation of the use of NC for increasing signal intensity and normalising sample acquisition by increasing spot homogeneity. The conclusion from this work was that $\mathrm{NC}$ improved reproducibility and ion signal intensity across a range of peptides and proteins. The results from bradykinin suggested a potential application of $\mathrm{NC}$ for quantitative workflows.

Two years later Liu et al(Liu et al. 1995) reported the use of NC for the analyses of DNA molecules. While their workflows were very similar to previous work, they offered a novel explanation for the physiochemical interactions occurring between the NC and the analyte. The DNA was reported as unable to interact with the NC due to its overall negative charge (this is shared by the NC). The NC therefore acted as a means to remove metal ion adducts from the DNA and purify it by binding these contaminants rather than the target molecules. It was also found that the NC amplified signal and increased spot homogeneity in a similar way to protein and peptide samples.

In 1997 Kussmann et al(Kussmann et al. 1997) employed NC and non-NC protocols to map the peptides of a neuron. It was found that ionisation efficiency of the sample was comparable with the $\mathrm{NC}$ and non-NC spots, with no greater difference found between the two. This stands in the face of an overwhelming body of literature that states otherwise, and it should be noted that no direct controlled comparison of NC and non-NC spots were made. The next year they followed up this work with a comprehensive investigation of NC preparation methodologies using modifications of thin and thick layer techniques published in the early 90's. It was found that the thin layer method was more sensitive for peptide samples with low levels of contamination whereas the thick layer proved to have a much greater capacity to absorb contaminants thereby significantly improving ion yields and the quality of subsequent spectra. 
An alternative method of achieving homogeneity was proposed by Landry et al in 2000(Landry, Lombardo and Smith 2000) this utilised modified methods that followed on from earlier work using thin film and fast evaporation methods, dubbed the Solution Phase Nitrocellulose method. It was found that allowing the matrix, nitrocellulose and sample to interact in the liquid phase facilitated the rapid evaporation of the organic solvent leaving a homogenous matrix and sample coating. The interaction between the nitrocellulose and sample was also reported to yield a 6 to 50 fold increase in sample intensity, though this may be a function of the dilution caused by premixing the sample and NC matrix solution.

Another publication in 2000 by Miliotis et al(Miliotis et al. 2000) used a modified thin coating method for pre-seeding a MALDI target prior to sample deposition. This method was adapted for use with the output from an autosampler equipped HPLC as opposed to individually spotted samples. The use of NC is reported to dramatically increase the homogeneity of matrix coverage and therefore the reproducibility of the method. This was crucial as this approach was designed to be a completely automated method for the spotting and subsequent analysis of chromatographic fractions via MALDI. It was also mentioned that $\mathrm{NC}$ can be used to facilitate washing of samples. The mechanism pertaining to how this is achieved is not mentioned nor is this performed in the paper. This work was expanded upon in 2002 when Miliotis et al(Miliotis et al. 2002) published a second paper that explored the use of NC substrates for LC-MALDI applications. There was a strong reported increase in $\mathrm{S} / \mathrm{N}$ however the increase in signal to noise is attributed to the prevention of the spot spreading, not to any physiochemical properties of the NC. It was found a coating of no more than $0.5 \mathrm{mg} / \mathrm{ml}$ of NC was ideal for maximising the ionisation efficiency of the sample. Since spot size was deemed the single most important factor, the sample viscosity and evaporation rate were treated as variables that directly affect the size of the spot. By controlling these two variables, spot size could also be controlled enabling the hydrophobic surface of the NC to concentrate the sample within each spot, thereby acting as a faux enrichment. The rationale behind this differs frompreviously published works as it does not acknowledge any properties of the NC beyond its hydrophobicity.

In 2004 Zhao et al(Zhao, Barber-Singh and Shippy 2004) published a paper aimed at comparing different application types of $\mathrm{NC}$ by comparing a modified version of the dried droplet technique with the thin film method. They aimed to determine which protocol was most compatible with desalting washes. Higher concentrations of $\mathrm{NC}$ at $20 \mathrm{mg} / \mathrm{ml}$ were used for the droplet method whereas only 5 $\mathrm{mg} / \mathrm{ml}$ of NC was used for the thin film method. Samples were also applied differently to traditional preparations; $\sim 1 \mu$ l of sample was pipetted onto the dried NC matrix and allowed to incubate for 3 minutes. The remaining liquid was then removed with the pipette. The reasoning behind this was that the incubation time in the liquid phase allowed the peptides to bind to the NC matrix mix, while the soluble salts remained in solution. The samples were then subsequently washed with $0.1 \%$ Trifluroacetic Acid (TFA) to acidify the spots, remove any residual salts and increase the S/N. A variation of this was also performed whereby 6 separate applications of the dilute peptide mix were performed consecutively. This was found to slowly deplete both the NC and matrix through dissolution resulting in lower overall signal intensity.

The hydrophobic properties of NC were also reported by Donegan et al in 2004(Donegan et al. 2004) however it was also reported that the presence of thin film NC suppressed matrix ions when performing analyses in the sub 500 Da mass range. The thin layer of matrix and NC was then deemed to have a dual effect of concentrating the sample into a very small area and supressing the incidence of MALDI matrix peaks in the subsequent generated spectra. This allowed peptides in a very low mass range, 150-500 $\mathrm{Da}$, to be analysed without the interference from matrix peaks. The proposed mechanism for this was that the dissolution of matrix and $\mathrm{NC}$ with the analyte creates an ideal analyte 
to matrix ratio thereby eliminating the incidence of matrix peaks. This phenomenon has been discussed elsewhere(Gobom et al. 2001).

Pang et al(Pang et al. 2004), also in 2004, reported that the interaction between NC and protein/peptide molecules was in fact electrostatic and that the differing concentrations of NC could in fact suppress ionisation due to the strong binding affinity of the high concentration NC. It was reported that the addition of $\mathrm{NC}$ allowed for the creation of homogenous seed layers of matrix crystals. This then allowed for uniform co-crystallisation of the matrix and analyte. It was also reported that adding $\mathrm{NC}$ at a concentration of between 0.1 and $1 \%$ significantly increased the ionisation efficiency of small molecular weight peptides $(<600 \mathrm{Da})$ while significantly increasing shot to shot reproducibility; less than this was unable to form a homogenous thin film, while more suppressed ionisation. It was proposed that the higher concentrations $(>1 \%)$ have a strong electrostatic binding affinity for the sample thereby preventing its effective ionisation.

Further to the work produced by Miliotis et al in 2002(Miliotis et al. 2002), Chen et al in 2005(Chen et al. 2005) expanded the pre coating protocol. The addition of NC to the MALDI target was chosen to create a uniform surface that allowed for the uniform continued deposition of the sample and crystallisation of the sample output from the chromatograph (figure 3). It should be noted that the mechanism responsible for this was described as hydrophilic interaction between the LC fractions and the NC. The uniformity of the streaking prevented the overlapping of LC fractions thereby increasing the dynamic range of the analysis. This interaction is described as hydrophilic which is in sharp contrast to every other paper discussed in this review and the reasoning for this is not postulated nor are any confirmatory experiments performed to support this explanation. There are also additional implications should this interaction be hydrophilic not hydrophobic. i.e ability to bind lipids or proteins/peptides in solutions containing high levels of organic solvents .

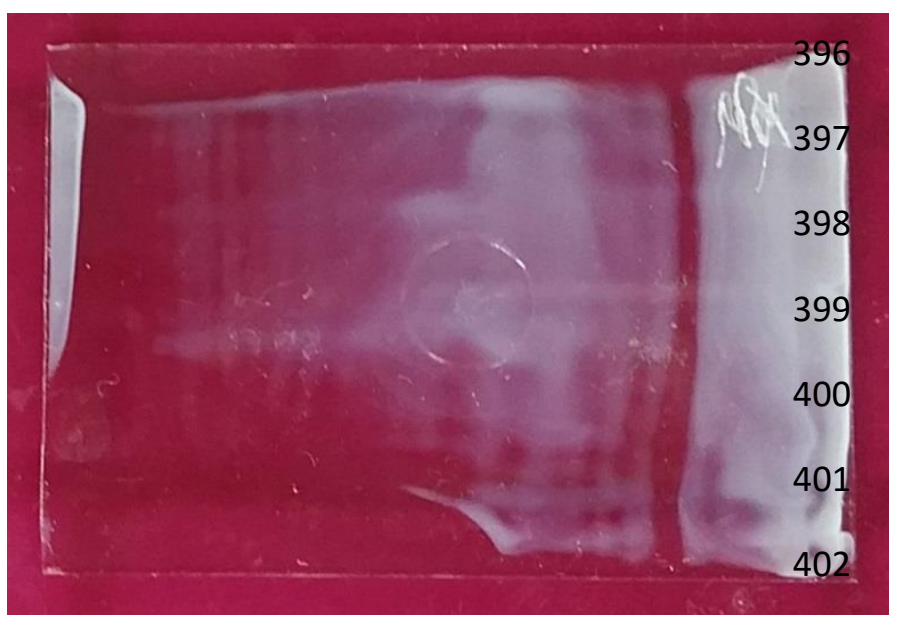

Figure 3. A Nitrocellulose coated glass Indium Tin Oxide (ITO) slide. Slides like this can be prepared from liquid NC and then used in LC MALDI or as a tissue fixative in IMS applications. The NC is smeared from right to left with another glass slide much to create a flat homogenous surface.

Luque-Garcia et al(Luque-Garcia et al. 2006) in 2006 created a modified western blot protocol that allowed for the analyses of proteins that had first been electro-blotted onto NC membrane. Once blotted, the portion of NC containing the sample of interest, was excised, dissolved in an appropriate solvent (see table 1.), trypsin digested and analysed via MALDI. It was found that use of electroblotting and liquid phase digestion, as opposed to in gel trypsin digestion, was faster and more sensitive with a reduction in time from 16 to 6 hours. By using this protocol the team was able to discern the molecular weight of two membrane bound proteins. It should be noted that there was no 
mention of the reported ionisation efficiency, homogeneity or reproducibility aspects of NC preparation and use.

In a continuation of the work by Shevchenko et al(Shevchenko et al. 1996) and Landry et al(Landry, Lombardo and Smith 2000), Wu et al(Wu, Hsieh and Tam 2006) published a modified protocol that described NC application to AnchorChips (Bruker Daltonics) that have already been prepared with hydrophilic spots surrounded by a hydrophobic barrier. The reported results confirm the findings of previous papers and describe an increase in signal intensity, homogeneity of spots and detection of additional peaks not found in non-NC samples. The researchers also report that the addition of NC increased the mass accuracy of the detected peaks when compared to previous papers that did not employ NC in their workflows. This is the first time that improved mass accuracy has been reported as a property afforded by the use of $\mathrm{NC}$ in mass spectrometry. Another AnchorChip preparation was performed by Kouvonan et al in 2009(Kouvonen et al. 2009). This work carries on from the initial work performed by Donegan et al(Donegan et al. 2004) however, it is the first to coin the term "Nitromatrix". The researchers found that the nitromatrix increased sequence coverage of proteins, the number of peptides detected, the Mascot scores of the detected peptides and also provided a resilient crystal layer that could withstand multiple analyses with the mass spectrometer. This was confirmed by multiple passes of the sample in imaging mode. This resilience was used to demonstrate that 10 sequential analyses of a single chromatographic run could be performed, resulting in the collection of over 15 million high quality MS spectra.

The most recent application of $\mathrm{NC}$ in a biomedical application was applied to a tissue imaging protocol by O'Rourke et al(O'Rourke, Djordjevic and Padula 2015). It was reported that the addition of NC served to fix tissue sections to the surface of the glass slides, allowing for repeated washing in a range of solvents and fixatives without any disruption to the structure of the tissue.

\section{Current consensus of effectiveness of methodologies}

The avenues of investigation that have been followed in the quest for finding reproducibility in MALDI are broad and varied. Initial methods of analysis with solid matrix powder proved to be unreliable resulting in heterogeneous coverage and inconsistent signal. The initial approaches that were undertaken to fix this were centered around ways of increasing the homogeneity of matrix crystal coverage and ensuring an even incorporation of the matrix and analyte during recrystallisation. Additional approaches utilising novel matrices as well as additives and specially prepared MALDI target plates have all been used as ways of standardising the process. Despite the sizeable body of literature that explores and proposes various methods that improve reproducibility the rate of uptake of any individual technique has not been particularly strong.

The use of ionic liqud matrices is a good example of the above whereby several papers have demonstrated its effectiveness in ensuring homogeneity with a pipetted sample. However, ionic liquid matrices have not become a ubiquitous technique indicating that either these alternative methods are not highly effective when put into routine use, or the time and financial expense involved with either purchasing the matrix commercially or synthesising it is simply too high for the limited benefit that would be gained. The use of specially prepared MALDI target plates is another example of this whereby commercially prepared single use plates can be expensive as a constant consumable and the time required to create and standardise the creation of them in a laboratory, on an individual basis, is too time-consuming to be worthwhile. 
It should also be noted that despite a substantial publication record, the utilisation of inorganic matrices as effective replacements in MALDI has also not shown to have a high uptake. Furthermore the lack of consensus between publications as to the ideal inorganic compounds that could be used for the analysis of small molecules such as metabolites and drugs, shows that this avenue of research has not been fully explored and would require substantial improvement to be truly effective, possible suggesting that other avenues have proven more fruitful. With this in mind, there is a single compound that has a large publication history and a clear potential as a universal preparation protocol; the utilisation of nitrocellulose.

There is a very clear body of evidence that supports the multitude of properties that NC appears to possess however, as can be seen from the earlier section, the mechanisms that are proposed to explain these properties are not in agreement and, unlike nanoflow chromatography coupled to nanoelectrospray ionisation, no commonalities in methods are evident. A perfect example is the ability to increase signal to noise in spotted samples. This has been attributed to: the hydrophobicity of the NC allowing the samples to concentrate; the reduction of matrix ions that cause ion suppression; the absorption of metal adducts and an ability of the NC to increase signal intensity when incorporated into the sample and matrix. Each of these proposed mechanisms could have implications when a research team is deciding whether NC possesses the properties needed for a specific workflow. Therefore it is of great importance for these physiochemical interactions to be studied in detail.

The lack of consensus in any methodology is detrimental to the larger body of literature. Conflicting reports pertaining to chemical mechanisms or interactions make informed experimentation by third parties difficult as there is no definitive evidence that one mechanism is more or less appropriate when compared to others. Another example of this is the description of $\mathrm{NC}$ possessing a highly hydrophobic quality. Every paper mentioned in this review agrees that $\mathrm{NC}$ is hydrophobic by nature. However Chen et al(Chen et al. 2005) state quite clearly that the hydrophilic nature of $\mathrm{NC}$ is what allows the uniform streaking of chromatograph eluent from their custom micro dispenser. Disagreement such as this, needs to be resolved in order to progress the use of this technique in the field of MALDI mass spectrometry.

There is also a level of disagreement when referring to the method used to prepare the nitrocellulose. As stated in section 2.2, "properties of nitrocellulose", there are a number of different preparation solutions as well as a number of different application methods that have been employed over the last 30 years. Table 1 provides a comprehensive list of methodological papers that have proposed variations of methods and solutions for the preparation of NC. It is very clear that the "ideal" recipe for the preparation of NC has not yet been discerned. Some parameters such as concentration of NC show a level of consensus i.e between 5 and $10 \mathrm{mg} / \mathrm{ml}$. However, solvent choice or the need to acidify the spots prior to analysis has not been agreed on.

There is also little agreement as to the application method that is most appropriate. Seeding the MALDI target with NC and matrix has been proposed as ideal, as has thin film coatings, thick film coatings and preparations that mix the sample matrix and $\mathrm{NC}$ together before spotting onto the MALDI target. There has been no definitive agreement regarding how the NC should be incorporated into a sample.

Finally there are some publications that have begun to employ NC in standard workflows without any description as to why. Shevchenko et al(Shevchenko et al. 1996) introduced NC into their standard MALDI preparation. There is no description as to why NC was incorporated into the matrix however, 
with the inclusion of a washing step; it can be assumed that it was used for its ability to capture protein. The adoption of these methodologies without proper investigation can be potentially damaging as unknown variables could arise without the attention of the investigator.

It is for all the above reasons that a concise and highly accurate investigation of the true properties of $\mathrm{NC}$ and their subsequent mechanisms is necessary.

\section{The application of low cost robotics and microfluidics to MALDI.}

It could be argued that the main reason for poor spot homogeneity is that the majority of sample handling for MALDI MS is done manually by a researcher with a handheld pipette. While there is no published direct evidence for this, logic and experience in other areas using automated liquid handling would suggest improvements could be made by automating sample application. The main impediment to this is cost as the currently available spotting robots cost more than $\$ 20,000$. The additional impediment is that commercially available systems allow minimal modification to their operation and are constructed of proprietary parts that are difficult to modify, preventing their adaption to the spotting methods described in section 2.1.

The last few years have seen great advances in electronics and rapid prototyping driven by the 'Maker Community'. Individuals or groups are able to use cheap microcontrollers, such as Arduino, or more sophisticated but still cheap single board computers, such as Raspberry Pi, to create sophisticated robotics. This is of immediate attraction to researchers as these open source platforms are easily adapted to the creation of scientific instrumentation, such as thermocyclers(Kalaitzis et al. 2015) and on-line liquid-liquid extraction(Hsieh, Liu and Urban 2015). Of immediate relevance to this review is the work of Stoeckli and Stabb(Stoeckli and Staab 2015) who have created a matrix deposition device for imaging mass spectrometry (iMatrixSpray, http://imatrixspray.com) from easily sourced and cost effective parts. The device is reported to spray in a reproducible manner when analysing pixel intensities after matrix spraying. The open source nature of this instrument means that it is 'hackable' or able to be modified to suit other purposes. In the context of this review, the purpose could be the repeatable spraying of matrix solutions but we also envisage that the device is able to be adapted as a spotter for nanoflow chromatography or for the electrospraying of samples. Moravcova et al (Moravcová et al. 2009) previously demonstrated reproducible chromatography using an S-shaped gradient generated in a single syringe, but a simple binary gradient nanoflow chromatograph could also be created using a microcontroller, two stepper motors and two syringes capable of high pressure operation. In our laboratory, we are currently recycling the stepper motor driven syringe pumps from a 20 year old SMART system (Pharmacia) to be controlled by an Arduino microcontroller.

The increased demand by the Maker community for miniaturised controllers, motors and sensors capable of a wide range of measurements is making available an array of low cost devices of great usefulness to researchers. The application of this technology to the issues outlined in this review could provide much needed solutions.

\section{X: Post-Acquisition processing of spectra to enhance signal.}

It is common practice when using MALDI to increase signal by simply increasing the number of laser shots taken, generating numerous sub-spectra which are then averaged into the final 'observed' spectra shown by the instrument control software. However, as pointed out in this review, it is widely recognised that there is extensive variability in spectra acquired from multiple acquisitions the same sample and this can lead to inconsistency in the repeated observation of peaks (Olson et al, 2008). 
This review has focused on pre-acquisition methodologies of improving the reproducibility of peak observation, but it should be noted that there are a number of post-acquisition methodologies that aim to perform the same task. These methods employ automated tests and algorithms to resample spectra (Malyarenko et al, 2006), evaluate replicate spectra (Olson et al, 2008 and 2011, Dekker et al, 2005) or sub-spectra (Meuleman et al, 2009), generate a consensus spectrum and report the consistent features with a statistical confidence interval attached even, in the case of some algorithms, if the spectra are from different sources or instruments (Olson et al, 2008 and 2011). These methods need to be distinguished from algorithms that look for correlation between spectra to determine differences that are suspected of being diagnostic of disease or a condition or algorithms that assess spectral quality prior to database searching (Yun et al, 2009).

While repeated acquisitions and post-acquisition generation of merged and consensus spectra can be performed on peptides and proteins, the post-acquisition processing of imaging MS data poses unique challenges. Imaging MS samples the molecular content at a fixed, discreet point, consuming the majority of the sample before moving to the next discreet point, the acquisition of replicate spectra is impossible or at best highly improbable. Therefore, much of the literature on post-acquisition processing has focused on normalisation to remove systematic artifacts affecting peak intensity, often occurring as a function of time, as ion transmission decreases over the extended acquisition times used in MALDI imaging (Deininger eal al, 2011).

The data generated through IMS analysis is designed to be viewed visually. This means that the numerical data must be converted accordingly which relies on the use of data normalisation. This simple process allows elements such as peak width and minor variation in surface height of a sample, to be accounted for numerically, creating consistent images. In our experience, since MALDI relies on TOF analysis, if a tissue section is not completely flat a "ghosting" effect can be observed whereby there is a shift in mass and intensity from one end of a tissue section to the other (usually $~ 5 \mathrm{Da}$ in mass) (figure 4). The easiest way to deal with this is already present in the processing software and works by the grouping the mass range of the entire width of a particular peak to form a single mass "group". This normalises the reported M/z by adding all mass points and creates a consistent and even image. This new normalised image then ensures that visual regions of high and low intensity are the result of actual molecule abundance and not a result of calibration or sample topography. We have used this method ourselves when generating data for our earlier MALDI IMS work and can attest to its function(O'Rourke, Djordjevic and Padula 2015). Other more complicated algorithmic techniques also exist such as the curation of data according to Gaussian distributions and the adherence to theoretical models to account for a lack of shot to shot reproducibility inherent to IMS(Widlak et al. 2016).

These post-acquisition methodologies do not negate the need to optimise pre-acquisition sample preparation steps however they can provide a further level of confidence that results observed are a complete and reproducible picture of the sample.

(1)

(1)

(1) 

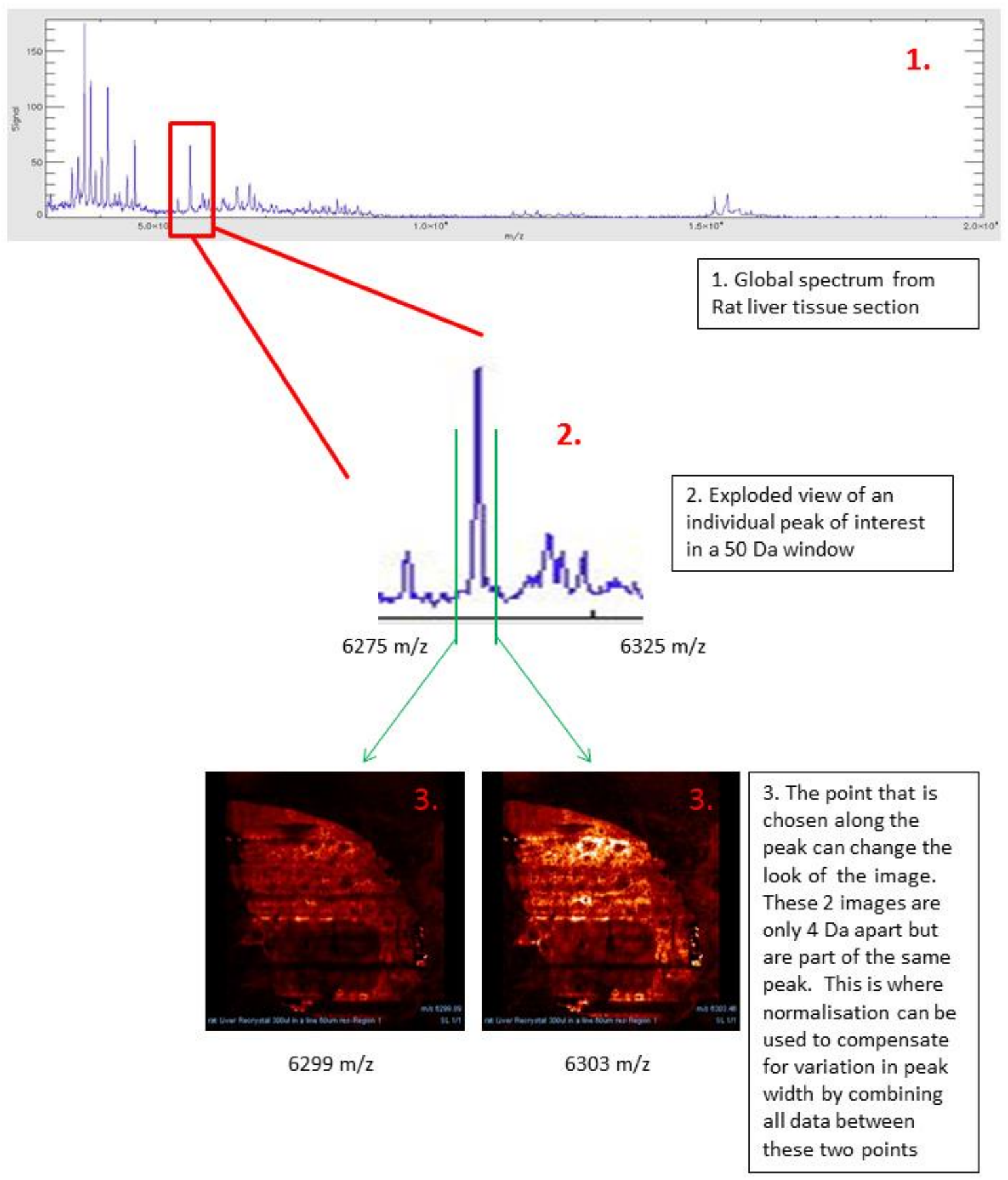

Figure 4. Flow diagram describing peak shift differences in IMS data from rat liver: The image on the left (3) displays the intensity of 6299 Da in the sample, or the left green line in the spectra (2), while the right image displays the intensity of $6303 \mathrm{Da}$, or the right green line in the spectra. It can clearly be seen that image intensity and macrostructure can change depending on where along the peak width the image is taken at. This data can be combined to form composite images

\section{Conclusion}

589 The pursuit of the ideal sample preparation methodology for MALDI is a topic of particular interest to 590 any and all MALDI mass spectrometrists. The rapid nature of MALDI makes it an ideal candidate for high throughput automated sample analysis of complex mixtures and purified proteins, with the 
592 ability to decouple Nano-flow liquid chromatography from mass spectrometry enabling less mass 593 spectrometer 'downtime' while waiting for column washes, sample loading and other blocks of time 594 where the MS is waiting for molecules to elute from chromatography. However, this cannot occur 595 until a robust and reproducible protocol for sample spotting and recrystallisation, which allows for 596 automated sample acquisition in both quantitative and qualitative analyses, is developed and broadly 597 adopted. In our opinion, the most promising candidate for such a protocol is NC.

598 The reported properties of NC are varied and potentially very useful when applied to MALDI mass 599 spectrometry. The creation of a universal protocol for the incorporation of NC to MALDI preparation 600 protocols could serve to fix the biggest issues associated with MALDI; increasing signal to noise, spot 601 homogeneity, reproducibility and reducing contaminants would make MALDI an even more powerful 602 analytical tool. It is for this reason that further research into NC should be made a priority for any 603 MALDI mass spectrometry based research laboratory. 


\begin{tabular}{|c|c|c|c|}
\hline \multicolumn{4}{|c|}{ Table 1. Nitrocellulose methodological publications } \\
\hline Paper & Year & Solvents used & Key methodological notes \\
\hline $\begin{array}{l}\text { M. Armand Ruffer, and M. } \\
\text { Crendiropoulo(Ruffer and } \\
\text { Crendiropoulo 1901) }\end{array}$ & 1901 & Unknown & $\begin{array}{l}\text { Unmodified NC membrane was used to fashion a 'sack' that was then used as a } \\
\text { semi-permeable membrane for the dialyses of enterotoxins from bacterial broth } \\
\text { culture. }\end{array}$ \\
\hline $\begin{array}{l}\text { William Brown(Brown } \\
\text { 1915) }\end{array}$ & 1915 & Ethanol, ether and camphor oil & $\begin{array}{l}\text { NC membranes with varying permeability's were produced from the addition of a } \\
\text { range of organic solvents and oils to liquid NC. They were the used for a number } \\
\text { of dialysis methodologies such as extraction of toxins from broth media. }\end{array}$ \\
\hline $\begin{array}{l}\text { Jonsson et al(Jonsson et al. } \\
\text { 1986) }\end{array}$ & 1986 & Amyl acetate & $\begin{array}{l}1 \% \mathrm{NC} \text { in amyl acetate was used to 'capture' proteins and peptides and allowed } \\
\text { washing to be performed with } 1 \mathrm{ml} \text { of milliQ water. }\end{array}$ \\
\hline Wilk et al(Wilk et al. 1988) & 1988 & $\begin{array}{l}\text { Amyl acetate, diluted with } \\
\text { methanol }\end{array}$ & $\begin{array}{l}\text { NC prepared as stock in amyl acetate and then diluted with methanol. The sample } \\
\text { was then added and the subsequent mix was spotted onto a target plate. }\end{array}$ \\
\hline $\begin{array}{l}\text { Mock et al(Mock, Sutton } \\
\text { and Cottrell 1992) }\end{array}$ & 1992 & Acetone & $\begin{array}{l}\text { NC was spotted onto the surface of a gold plated MALDI target with a modified } \\
\text { electrospray apparatus followed by addition of sample. MilliQ water was used to } \\
\text { wash the sample to remove soluble contaminants then } 0.5 \mu \text { l of matrix was } \\
\text { overlayed. The matrix was not allowed to dry immediately, facilitating the } \\
\text { extraction of protein off the surface of the NC*. }\end{array}$ \\
\hline $\begin{array}{l}\text { Preston et al(Preston, } \\
\text { Murray and Russell 1993) }\end{array}$ & 1993 & Methanol & $\begin{array}{l}\text { NC was spotted onto the surface of a MALDI target plate, allowed to dry, then } \\
\text { overlayed with sample and matrix. }\end{array}$ \\
\hline Liu et al(Liu et al. 1995) & 1995 & Methanol, acetone and acetonitrile & $\begin{array}{l}\text { NC was dissolved in a number of solvents and used as a substrate for DNA } \\
\text { analysis in MALDI. }\end{array}$ \\
\hline $\begin{array}{l}\text { Shevchenko(Shevchenko et } \\
\text { al. 1996) }\end{array}$ & 1996 & Acetone, 2-proponal & $\begin{array}{l}\text { Nitrocellulose is introduced into the matrix and deposited onto the MALDI target } \\
\text { plate. The sample is overlayed and then washed with MilliQ water. }\end{array}$ \\
\hline $\begin{array}{l}\text { Kussmann et al(Kussmann } \\
\text { et al. 1997) }\end{array}$ & 1997 & $\begin{array}{l}\text { Acetone then diluted with } \\
\text { isopropanol }\end{array}$ & $\begin{array}{l}\text { NC was applied to the target plate using spin coating, spraying and transferred on a } \\
\text { piece of scotch tape. Both the dried droplet and sandwich methods were used when } \\
\text { applying sample and matrix to the NC spots. }\end{array}$ \\
\hline $\begin{array}{l}\text { Kussmann et al(Kussmann } \\
\text { et al. 1997) }\end{array}$ & 1997 & $\begin{array}{l}\text { Acetone then diluted with propan- } \\
2-\mathrm{ol}\end{array}$ & $\begin{array}{l}\text { NC is first prepared in acetone the diluted with isopropanol. It is then spin coated } \\
\text { (thick layer technique) or mixed with matrix and allowed to dry (thin layer } \\
\text { technique) }\end{array}$ \\
\hline $\begin{array}{l}\text { Landry et al(Landry, } \\
\text { Lombardo and Smith 2000) }\end{array}$ & 2000 & Acetone and isopropanol & $\begin{array}{l}\text { A hybrid method was developed termed the "solution-phase nitrocellulose" } \\
\text { method. The sample and nitrocellulose are dissolved in acetone and TFA then this } \\
\text { matrix solution is mixed in a 1:1 ratio with sample, spotted onto the MALDI plate } \\
\text { and allowed to dry. }\end{array}$ \\
\hline
\end{tabular}

*See discussion for further details 


\begin{tabular}{|c|c|c|c|}
\hline $\begin{array}{l}\text { Miliotis et al(Miliotis et al. } \\
2000)\end{array}$ & 2000 & Acetone and isopropanol & $\begin{array}{l}5 \mathrm{mg} / \mathrm{ml} \text { of NC and } 20 \mathrm{mg} / \mathrm{ml} \text { matrix were sprayed with an airbrush onto the } \\
\text { surface of a stainless steel MALDI target. This provided an initial seed layer that } \\
\text { subsequent sample is then applied to via a piezoelectric micro-dispenser. }\end{array}$ \\
\hline $\begin{array}{l}\text { Miliotis et al(Miliotis et al. } \\
\text { 2002) }\end{array}$ & 2002 & Acetone and isopropanol & $\begin{array}{l}\text { NC and saturated matrix was used as a thin layer coating to prepare targets for } \\
\text { later sample deposition. An automated piezoelectric micro dispenser was then used } \\
\text { to spot sample directly onto the surface of the seed layer of matrix/NC. }\end{array}$ \\
\hline $\begin{array}{l}\text { Zhao et al(Zhao, Barber- } \\
\text { Singh and Shippy 2004) }\end{array}$ & 2004 & Acetone and isopropanol & $\begin{array}{l}\text { The thin film method of NC application was compared to a variation of the dried } \\
\text { droplet method whereby matrix and NC were combined and spotted onto the } \\
\text { surface of a MALDI plate prior to sample application. }\end{array}$ \\
\hline $\begin{array}{l}\text { Donegan et al(Donegan et } \\
\text { al. 2004) }\end{array}$ & 2004 & Acetone & $\begin{array}{l}\text { NC and matrix were co-mixed then applied to the surface of a hydrophobically } \\
\text { coated MALDI plate via spin coating. The sample in question as then spotted on to } \\
\text { the surface. }\end{array}$ \\
\hline Pang et al(Pang et al. 2004) & 2004 & $\begin{array}{l}\text { Acetone then diluted with } \\
\text { isopropanol }\end{array}$ & $\begin{array}{l}\text { Nitrocellulose solution was prepared in } 2 \%(\mathrm{~W} / \mathrm{V})(\text { equivalent to } 20 \mathrm{mg} / \mathrm{ml}) \\
\text { solution then diluted down to } 1.0 \%, 0.5 \%, 0.25 \%, 0.1 \%, 0.05 \% \text { solutions, spotted } \\
\text { onto the surface of a MALDI target and allowed to dry at ambient temperature. } \\
\text { The sample and matrix was then overlayed using the sandwich method }\end{array}$ \\
\hline $\begin{array}{l}\text { Chen et al(Chen et al. } \\
\text { 2005) }\end{array}$ & 2005 & Acetone & $\begin{array}{l}\text { NC solution was prepared in acetone then applied via spin coating to the surface of } \\
\text { a MALDI target plate a custom LC streaking apparatus was then used to apply } \\
\text { sample in a serpentine way to the NC film. }\end{array}$ \\
\hline $\begin{array}{l}\text { Luque-Garcia et al(Luque- } \\
\text { Garcia et al. 2006) }\end{array}$ & 2006 & Acetone, methanol, acetonitrile & $\begin{array}{l}\text { Proteins were electro blotted from 1D SDS-PAGE gels onto NC membrane. The } \\
\text { band on the membrane were then cut out and dissolved in matrix solution } \\
\text { containing an appropriate solvent. The dissolved spots were then spotted onto a } \\
\text { stainless steel MALDI target and allowed to dry before direct analysis. }\end{array}$ \\
\hline $\begin{array}{c}\text { Wu et al(Wu, Hsieh and } \\
\text { Tam 2006) }\end{array}$ & 2006 & $\begin{array}{c}\text { Acetone, isopropanol in a } 7: 2 \mathrm{v}: \mathrm{v} \\
\text { ratio }\end{array}$ & $\begin{array}{l}\text { Preparation of NC was performed in line with the protocols from Shevchenko et } \\
\text { al(Shevchenko et al. 1996) and Landry et al(Landry, Lombardo and Smith 2000). } \\
\text { However, instead of MALDI target plates, NC was spotted onto AnchorChip } \\
\text { plates prior to sample deposition. }\end{array}$ \\
\hline $\begin{array}{c}\text { Kouvonan et al(Kouvonen } \\
\text { et al. 2009) }\end{array}$ & 2009 & $\begin{array}{c}\text { Acetone, acetonitrile, isopropanol, } \\
0.1 \% \text { TFA in a } 7: 7: 2: 2 \mathrm{v}: \mathrm{v} \text { ratio }\end{array}$ & $\begin{array}{l}\text { A peptide sample was spotted onto the surface of an AnchorChip and then } \\
\text { overlayed with NC and matrix combined together (coined as 'Nitromatrix' in a } \\
\text { complex solvent mix). This nitromatrix was then compared to non-NC matrix } \\
\text { application and the standard PAC protocol for AnchorChip preparation }\end{array}$ \\
\hline $\begin{array}{l}\text { O’Rourke et al(O'Rourke, } \\
\text { Djordjevic and Padula } \\
\text { 2015) }\end{array}$ & 2015 & Acetone & $\begin{array}{l}\text { NC was prepared at a concentration of } 40 \mathrm{mg} / \mathrm{ml} \text { and applied to ITO coated glass } \\
\text { slides using the method commonly employed for preparing blood smears. }\end{array}$ \\
\hline
\end{tabular}


Armstrong, D. W., et al. (2001). "lonic Liquids as Matrixes for Matrix-Assisted Laser Desorption/Ionization Mass Spectrometry." Anal Chem 73(15): 3679-3686.

Axelsson, J., et al. (1997). "Improved Reproducibility and Increased Signal Intensity in Matrixassisted Laser Desorption/Ionization as a Result of Electrospray Sample Preparation." Rapid Communications in Mass Spectrometry 11(2): 209-213.

Bahr, U., Karas, M. and Hillenkamp, F. (1994). "Analysis of biopolymers by matrix-assisted laser desorption/ionization (MALDI) mass spectrometry." Fresenius' Journal of Analytical Chemistry 348(12): 783-791.

Bernier, M. C., Wysocki, V. H. and Dagan, S. (2015). "Laser desorption ionization of small molecules assisted by tungsten oxide and rhenium oxide particles." J Mass Spectrom 50(7): 891-898.

Boom, D. v. d., et al. (2004). "MALDI-TOF MS: a platform technology for genetic discovery." International Journal of Mass Spectrometry 238(2): 173-188.

Brown, W. (1915). "On the Preparation of Collodion Membranes of Differential Permeability." Biochem J 9(4): 591-617.

Brown, W. (1915). "Preliminary Note on the Preparation of Collodion Dialysers of Graded Permeability." Biochem J 9(3): 320.

Bruker (2013). "Sinapinic Acid Instructions for use Revision 2."

Castro, A. L., et al. (2008). "Titanium dioxide anatase as matrix for matrix-assisted laser desorption/ionization analysis of small molecules." Rapid Commun Mass Spectrom 22(23): 37613766.

Chen, H. S., et al. (2005). "High-speed, high-resolution monolithic capillary LC-MALDI MS using an off-line continuous deposition interface for proteomic analysis." Anal Chem 77(8): 2323-2331.

Chughtai, K. and Heeren, R. M. (2010). "Mass spectrometric imaging for biomedical tissue analysis." Chem Rev 110(5): 3237-3277.

Cohen, S. L. and Chait, B. T. (1996). "Influence of matrix solution conditions on the MALDI-MS analysis of peptides and proteins." Anal Chem 68(1): 31-37.

Donegan, M., et al. (2004). "Controlling matrix suppression for matrix-assisted laser desorption/ionization analysis of small molecules." Rapid Communications in Mass Spectrometry 18(17): 1885-1888.

Dong, X., et al. (2010). "Graphene as a novel matrix for the analysis of small molecules by MALDITOF MS." Anal Chem 82(14): 6208-6214.

Enthaler, B., et al. (2013). "MALDI imaging in human skin tissue sections: focus on various matrices and enzymes." Anal Bioanal Chem 405(4): 1159-1170.

Fleith, C., et al. (2014). "Laser desorption ionization mass spectrometry of peptides on a hybrid CHCA organic-inorganic matrix." Analyst 139(15): 3748-3754.

Fountoulakis, M. and Langen, H. (1997). "Identification of proteins by matrix-assisted laser desorption ionization-mass spectrometry following in-gel digestion in low-salt, nonvolatile buffer and simplified peptide recovery." Anal Biochem 250(2): 153-156.

Francese, S., et al. (2013). "Curcumin: a multipurpose matrix for MALDI mass spectrometry imaging applications." Anal Chem 85(10): 5240-5248.

Friesen, W. L., et al. (2015). "Two-dimensional graphene as a matrix for MALDI imaging mass spectrometry." J Am Soc Mass Spectrom 26(11): 1963-1966.

Fukuyama, Y. (2015). "MALDI Matrix Research for Biopolymers." Mass Spectrom (Tokyo) 4(1): A0037.

Gobom, J., et al. (2001). " $\alpha$-Cyano-4-hydroxycinnamic Acid Affinity Sample Preparation. A Protocol for MALDI-MS Peptide Analysis in Proteomics." Anal Chem 73(3): 434-438.

Hillenkamp, F., et al. (1991). "Matrix-assisted laser desorption/ionization mass spectrometry of biopolymers." Anal Chem 63(24): 1193A-1203A.

Hou, J., et al. (2014). "Organic salt NEDC (N-naphthylethylenediamine dihydrochloride) assisted laser desorption ionization mass spectrometry for identification of metal ions in real samples." Analyst 139(13): 3469-3475. 
Hsieh, K.-T., Liu, P.-H. and Urban, P. L. (2015). "Automated on-line liquid-liquid extraction system for temporal mass spectrometric analysis of dynamic samples." Anal Chim Acta 894: 35-43.

Jackson, S. N., et al. (2014). "MALDI-Ion Mobility Mass Spectrometry of Lipids in Negative Ion Mode." Analytical methods : advancing methods and applications 6(14): 5001-5007.

Jaskolla, T., et al. (2009). "The new matrix 4-chloro-alpha-cyanocinnamic acid allows the detection of phosphatidylethanolamine chloramines by MALDI-TOF mass spectrometry." J Am Soc Mass Spectrom 20(5): 867-874.

Jonsson, G. P., et al. (1986). "Plasma desorption mass spectrometry of peptides and proteins adsorbed on nitrocellulose." Anal Chem 58(6): 1084-1087.

Kalaitzis, P., et al. (2015). "A Rapid and Low-Cost PCR Thermal Cycler for Low Resource Settings." PLoS One 10(7): e0131701.

Kang, H. S., et al. (2011). "Protein and lipid MALDI profiles classify breast cancers according to the intrinsic subtype." BMC Cancer 11: 465.

Karas, M. and Hillenkamp, F. (1988). "Laser desorption ionization of proteins with molecular masses exceeding 10,000 daltons." Anal Chem 60(20): 2299-2301.

Kjellstrom, S. and Jensen, O. N. (2004). "Phosphoric acid as a matrix additive for MALDI MS analysis of phosphopeptides and phosphoproteins." Anal Chem 76(17): 5109-5117.

Knochenmuss, R. and Zenobi, R. (2003). "MALDI ionization: the role of in-plume processes." Chem Rev 103(2): 441-452.

Kouvonen, P., et al. (2009). "Nitromatrix provides improved LC-MALDI signals and more protein identifications." Proteomics 9(6): 1662-1671.

Krasny, L., Hynek, R. and Kodicek, M. (2011). "Disulfide bond decay during matrix-assisted laser desorption/ionization time-of-flight mass spectrometry experiments." Rapid Commun Mass Spectrom 25(17): 2468-2474.

Kruegel, A., Pavlov, J. and Attygalle, A. B. (2013). "Enhancement of laser desorption ionization mass spectrometric signals of cesium iodide by elemental sulfur." Rapid Commun Mass Spectrom 27(7): 763-766.

Kussmann, M., et al. (1997). "Matrix-assisted laser desorption/ionization mass spectrometric peptide mapping of the neural cell adhesion protein neurolin purified by sodium dodecyl sulfate polyacrylamide gel electrophoresis or acidic precipitation." J Mass Spectrom 32(5): 483-493.

Kussmann, M., et al. (1997). "Matrix-assisted Laser Desorption/Ionization Mass Spectrometry Sample Preparation Techniques Designed for Various Peptide and Protein Analytes." Journal of Mass Spectrometry 32(6): 593-601.

Landry, F., Lombardo, C. R. and Smith, J. W. (2000). "A method for application of samples to matrixassisted laser desorption ionization time-of-flight targets that enhances peptide detection." Anal Biochem 279(1): 1-8.

Li, L., Golding, R. E. and Whittal, R. M. (1996). "Analysis of Single Mammalian Cell Lysates by Mass Spectrometry." Journal of the american chemical society 118(46): 11662-11663.

Li, Y. L. and Gross, M. L. (2004). "Ionic-liquid matrices for quantitative analysis by MALDI-TOF mass spectrometry." J Am Soc Mass Spectrom 15(12): 1833-1837.

Liu, Y.-H., et al. (1995). "Use of a nitrocellulose film substrate in matrix-assisted laser desorption/ionization mass spectrometry for DNA mapping and screening." Anal Chem 67(19): 34823490.

Luque-Garcia, J. L., et al. (2006). "Use of nitrocellulose membranes for protein characterization by matrix-assisted laser desorption/ionization mass spectrometry." Anal Chem 78(14): 5102-5108.

Marsico, A. L., et al. (2015). "Inkjet-printed gold nanoparticle surfaces for the detection of low molecular weight biomolecules by laser desorption/ionization mass spectrometry." J Am Soc Mass Spectrom 26(11): 1931-1937.

Miliotis, T., et al. (2000). "Capillary liquid chromatography interfaced to matrix-assisted laser desorption/ionization time-of-flight mass spectrometry using an on-line coupled piezoelectric flowthrough microdispenser." Journal of Mass Spectrometry 35(3): 369-377. 
Miliotis, T., et al. (2002). "Ready-made matrix-assisted laser desorption/ionization target plates coated with thin matrix layer for automated sample deposition in high-density array format." Rapid Commun Mass Spectrom 16(2): 117-126.

Mock, K. K., Sutton, C. W. and Cottrell, J. S. (1992). "Sample immobilization protocols for matrixassisted laser-desorption mass spectrometry." Rapid Commun Mass Spectrom 6(4): 233-238.

Moravcová, D., et al. (2009). "Short monolithic columns for purification and fractionation of peptide samples for matrix-assisted laser desorption/ionization time-of-flight/time-of-flight mass spectrometry analysis in proteomics." Journal of Chromatography A 1216(17): 3629-3636. Nishikaze, T., et al. (2012). "Correlation between Sweet Spots of Glycopeptides and Polymorphism of the Matrix Crystal in MALDI Samples." Mass Spectrom (Tokyo) 1(1): A0006.

Nordhoff, E., Lehrach, H. and Gobom, J. (2007). "Exploring the limits and losses in MALDI sample preparation of attomole amounts of peptide mixtures." International Journal of Mass Spectrometry 268(2-3): 139-146.

O'Rourke, M. B., Djordjevic, S. P. and Padula, M. P. (2015). "A non-instrument-based method for the analysis of formalin-fixed paraffin-embedded human spinal cord via matrix-assisted laser desorption/ionisation imaging mass spectrometry." Rapid Communications in Mass Spectrometry 29(19): 1836-1840.

Onnerfjord, P., et al. (1999). "Homogeneous sample preparation for automated high throughput analysis with matrix-assisted laser desorption/ionisation time-of-flight mass spectrometry." Rapid Commun Mass Spectrom 13(5): 315-322.

Owen, S. J., et al. (2003). "Increasing sensitivity and decreasing spot size using an inexpensive, removable hydrophobic coating for matrix-assisted laser desorption/ionisation plates." Rapid Commun Mass Spectrom 17(21): 2439-2449.

Pang, R. T. K., et al. (2004). "Technical Evaluation of MALDI-TOF Mass Spectrometry for Quantitative Proteomic Profiling: Matrix Formulation and Application." Clin Proteomics 1(3-4): 259-270.

Preston, L. M., Murray, K. K. and Russell, D. H. (1993). "Reproducibility and quantitation of matrixassisted laser desorption ionization mass spectrometry: effects of nitrocellulose on peptide ion yields." Biol Mass Spectrom 22(9): 544-550.

Ruffer, M. A. and Crendiropoulo, M. (1901). "Note on the Dialysis of the Toxins through Collodion Walls." Br Med J 1(2088): 14.

Sato, H., et al. (2011). "Ribosomal protein profiling by matrix-assisted laser desorption/ionization time-of-flight mass spectrometry for phylogenety-based subspecies resolution of Bifidobacterium longum." Syst Appl Microbiol 34(1): 76-80.

Sedo, O., Sedlacek, I. and Zdrahal, Z. (2011). "Sample preparation methods for MALDI-MS profiling of bacteria." Mass Spectrom Rev 30(3): 417-434.

Shan, Z., et al. (2007). "Mesoporous tungsten titanate as matrix for matrix-assisted laser desorption/ionization time-of-flight mass spectrometry analysis of biomolecules." Anal Chim Acta 593(1): 13-19.

Shevchenko, A., et al. (1996). "Mass Spectrometric Sequencing of Proteins from Silver-Stained Polyacrylamide Gels." Anal Chem 68(5): 850-858.

Stoeckli, M. and Staab, D. (2015). "Reproducible Matrix Deposition for MALDI MSI Based on OpenSource Software and Hardware." J Am Soc Mass Spectrom 26(6): 911-914.

Sun, G., et al. (2007). "Shotgun metabolomics approach for the analysis of negatively charged watersoluble cellular metabolites from mouse heart tissue." Anal Chem 79(17): 6629-6640.

Sunner, J., Dratz, E. and Chen, Y.-C. (1995). "Graphite surface-assisted laser desorption/ionization time-of-flight mass spectrometry of peptides and proteins from liquid solutions." Anal Chem 67(23): 4335-4342.

T-W. Dominic Chan, A. W. C., Peter J. Derrick, Derek J. Gardiner and Michael Bowden (1992). "Suppression of Matrix Ions in Ultraviolet Laser Desorption: Scanning Electron Microscopy and Rarnan Spectroscopy of the Solid Samples." Organic Mass spectrometry 27: 188-194. 
Tanaka, K., et al. (1988). "Protein and polymer analyses up tom/z 100000 by laser ionization timeof-flight mass spectrometry." Rapid Communications in Mass Spectrometry 2(8): 151-153.

Technology, H. F. (2010). " $\mu$ Focus Sample Plates for MALDI-TOF MS." 1-16.

Vaidyanathan, S. and Goodacre, R. (2007). "Quantitative detection of metabolites using matrixassisted laser desorption/ionization mass spectrometry with 9-aminoacridine as the matrix." Rapid Commun Mass Spectrom 21(13): 2072-2078.

Weaver, E. M. and Hummon, A. B. (2013). "Imaging mass spectrometry: From tissue sections to cell cultures." Adv Drug Deliv Rev 65(8): 1039-1055.

Widlak, P., et al. (2016). "Detection of molecular signatures of oral squamous cell carcinoma and normal epithelium - application of a novel methodology for unsupervised segmentation of imaging mass spectrometry data." Proteomics: n/a-n/a.

Wilk, Z. A., et al. (1988). "Factors affecting the quantitation of organic compounds in laser mass spectrometry." Anal Chem 60(21): 2338-2346.

Wu, Y. C., Hsieh, C. H. and Tam, M. F. (2006). "Matrix-assisted laser desorption/ionization of peptides on AnchorChip targets with alpha-cyano-4-hydroxycinnamic acid and nitrocellulose as matrix." Rapid Commun Mass Spectrom 20(2): 309-312.

Xiang, F. and Beavis, R. C. (1993). "Growing protein-doped sinapic acid crystals for laser desorption: An alternative preparation method for difficult samples." Organic Mass spectrometry 28(12): 14241429.

Zhao, X., Barber-Singh, J. and Shippy, S. A. (2004). "MALDI-TOF MS detection of dilute, volumelimited peptide samples with physiological salt levels." Analyst 129(9): 817-822.

Zhu, X. and Papayannopoulos, I. A. (2003). "Improvement in the detection of low concentration protein digests on a MALDI TOF/TOF workstation by reducing alpha-cyano-4-hydroxycinnamic acid adduct ions." J Biomol Tech 14(4): 298-307. 